\title{
Análise comparativa entre a quimioterapia e nanofibras de quitosana à frente de
}

\section{neoplasias malignas}

\author{
Comparative analysis between chemotherapy and chitosan nanofibers ahead of malignant \\ neoplasms
}

Analisis comparativo entre quimioterapia y nanofibras de quitosano frente a neoplasias malignas

Recebido: 07/02/2022 | Revisado: 14/02/2022 | Aceito: 16/02/2022 | Publicado: 24/02/2022

\author{
Érica Karine Alves de Lima \\ ORCID: https://orcid.org/0000-0003-1405-1960 \\ Universidade Federal do Piauí, Brasil \\ E-mail: ericakarinelima@gmail.com \\ Nágila Iane Pacheco \\ ORCID: https://orcid.org/0000-0002-2836-1639 \\ Centro Universitário UniFacid/Wyden, Brasil \\ E-mail: nagilaiane@hotmail.com \\ André Cardoso Tavares \\ ORCID: https://orcid.org/0000-0002-8413-3868 \\ Centro Universitário UniFacid/Wyden, Brasil \\ E-mail: andrebiomed16@gmail.com \\ Beatriz Lemos da Silva Loureiro \\ ORCID: https://orcid.org/0000-0002-9585-6586 \\ Centro Universitário UniFacid/Wyden, Brasil \\ E-mail: beatrizllms@outlook.com \\ Danielle Costa Lopes \\ ORCID: https://orcid.org/0000-0002-7382-1323 \\ Centro Universitário UniFacid/Wyden, Brasi \\ E-mail: dany197_@hotmail.com \\ Luiza Aragão Paiva Pires Ferreira Mendes \\ ORCID: https://orcid.org/0000-0002-4017-6102 \\ Centro Universitário UniFacid/Wyden, Brasil \\ E-mail: luizamendes0412@gmail.com
}

\begin{abstract}
Resumo
Introdução: O câncer (CA) é uma das doenças letais mais terríveis mundialmente e o sucesso de suas estratégias de tratamento quimioterápico atuais é limitado devido às várias desvantagens associadas, no entanto, os pesquisadores buscam meios de aprimorados materiais e produzir novos, neste estudo elaborou um levantamento bibliográfico sobre a utilização de nanofibras de quitosana como biopolímero no tratamento de neoplasias malignas. Resultados e Discursão: A quimioterapia tornou-se uma tática predominante para as majoritárias terapias, porém por possui muitos efeitos colaterais intensos, devido ao potencial promotor de apoptose, nas células cancerígenas e nas células normais, assim apresentando limitações inerentes, acarreta na busca de novas estratégias que solucione a tolerância de $\mathrm{pH}$, baixa solubilidade em água e difusão intracelular na entrega de fármacos, com isso, a quitosana demonstra-se biocompatibilidade e possuir a capacidade de liberação sustentada dos compostos anticancerígenos, por conseguir carregar fármacos com o $\mathrm{IC}^{50}$ reduzidos e agentes sintéticos, como 5-FU, aumenta a disponibilidade e eficácia contra o CA. Conclusão: Em suma, a aplicação da quitosana no tratamento quimioterápico contribui positivamente para sua eficácia e para amenizar seus efeitos colaterais.
\end{abstract}

Palavras-chave: Quitosana; Nanofibras; Câncer; Quimioterapia; Vantagem; Desvantagem.

\begin{abstract}
Introduction: Cancer (CA) is one of the most terrible lethal diseases worldwide and the success of their current chemotherapy treatment strategies is limited due to several associated disadvantages, however, the researchers look for ways to improve the materials and produce new ones, in this study. Developed a bibliographic survey on the use of chitosan nanofibers as a biopolymer in the treatment of malignant neoplasms. Results and Discussion: Chemotherapy has become a predominant tactic for the majority of therapies, , but because it has many intense side effects, due to the potential to promote apoptosis, in cancer cells and in normal cells, thus presenting inherent limitations, it leads to the search for new strategies that solve the $\mathrm{pH}$ tolerance, low water solubility and intracellular diffusion in the delivery of drugs, with this, the chitosan demonstrates biocompatibility and has the ability to release sustained anticancer compounds, by being able to load drugs with reduced IC50 and synthetic agents, such as 5-FU, increase availability
\end{abstract}


and effectiveness against CA. Conclusion: In short, the application of chitosan in chemotherapy contributes positively to its effectiveness and to alleviate its side effects.

Keywords: Chitosan; Nanofibers; Cancer; Chemotherapy; Advantage; Disadvantage.

\section{Resumen}

Introducción: El cáncer (CA) es una de las enfermedades letales más terribles a nivel mundial y el éxito de sus actuales estrategias de tratamiento con quimioterapia es limitado debido a varias desventajas asociadas, sin embargo, los investigadores buscan formas de mejorar los materiales y producir nuevos, en este sentido. Elaboró un levantamiento bibliográfico sobre el uso de nanofibras de quitosano como biopolímero en el tratamiento de neoplasias malignas. Resultados y Discusión: La quimioterapia se ha convertido en una táctica predominante para la mayoría de las terapias, pero debido a que tiene muchos efectos secundarios intensos, debido al potencial de promover la apoptosis, en células cancerosas y en células normales, presentando así limitaciones inherentes, conduce a la búsqueda de nuevas estrategias que resuelvan la tolerancia al $\mathrm{pH}$, la baja hidrosolubilidad y la difusión intracelular en la entrega de fármacos, con esto, la quitosa demuestra biocompatibilidad y tiene la capacidad de liberar compuestos anticancerígenos sostenidos, al poder cargar fármacos con IC50 y los agentes sintéticos, como el 5-FU, aumentan la disponibilidad y la eficacia contra la CA. Conclusión: En definitiva, La aplicación de quitosano em la quimioterapia contribuy e positivamente a su eficacia y a paliar sus efectos secundarios.

Palabras clave: Quitosano; Nanofibras; Cáncer; Quimioterapia; Ventaja; Desventaja.

\section{Introdução}

O câncer (CA) é uma das doenças letais mais terríveis mundialmente e o sucesso de suas estratégias de tratamento quimioterápico atuais é limitado devido a várias desvantagens associadas (Kamath, 2017; Jiang et al., 2021). Supõem que em 2040, com estilo de vida, elevação populacional e aumento na expectativa de idade, acarretam com a estimativa de, aproximadamente, 29 a 37 milhões conterá essa patologia (Jiang et al., 2021). Está baseia-se na inibição da divisão de células de crescimento rápido, característica das células cancerígenas, mas atingem, igualmente, as células normais com rápida proliferação, como os folículos pilosos, medula óssea e células do trato gastrointestinal, gerando os efeitos colaterais característicos da quimioterapia (Ashrafizadeh et al., 2021).

O tratamento usual é a quimioterapia, na forma monoterapia (medicamento de forma isolada) ou poliquimioterapia (com combinações), contendo como finalidade de desacelerar o processo de crescimento de células tumorais (Lima, 2019). Entretanto, os óbitos por doenças cancerígenas ainda são um problema de saúde pública que está em continuo acréscimo ao longo dos anos e se faz necessário o desenvolvimento de outras ferramentas ou aprimoramento de técnicas para ajudar na eficiência na terapia carcinogênica (Ashrafizadeh et al., 2021).

Atualmente, os pesquisadores buscam meios de aprimorar a sustentabilidade do meio ambiente, descobrindo novos materiais. Entre os mais variados utilizados, o polímero está se destacando por suas características físico-químicas. Devido a suas propriedades como: maior resistência a corrosão, flexibilidade, elasticidade, transparência, baixa densidade, baixa temperatura de processamento, baixa reatividade, entre outras propriedades (Silva, Rabelo, 2017). Nesse sentido, os polímeros advindos e fontes naturais vêm ganhando espaços por serem considerados sustentáveis, atóxicos, biocompatíveis e promitente por meio de nanomateriais, muitos utilizados em diversas aplicações, como eletroeletrônico, sensores e catalizadores. (Ali, Ahmed, 2018; Ashrafizadeh et al., 2021).

Um desses biopolímeros de origem natural é conhecido como quitina (C8H13O5N)n, um polissacarídeo com monômero de $\mathrm{N}$-acetilglucosamina, encontrada em paredes celulares de esponjas e fungos, filamentos de madeiras marinhas, crisálidas de bicho-da-seda, exoesqueletos de artrópodes como crustáceos e insetos e em escamas de peixes (Sudatta et al., 2020). A partir da sua desmineralização, descoloração, desacetilação, desproteinização, produz-se a quitosana (CS), sua forma desacetilada (Madni et al., 2021; Shafabakhsh et al., 2020).

A quitosana (CS) contém baixa toxicidade, biodegradabilidade, proveniente de meios renováveis, possui atividades antimicrobianas, compatibilidade, eficácia e baixa imunogenicidade, contendo seu uso para preparar cromatografia de afinidade, imobilizar enzimas, em alimentos e em biossensores (Ali, Ahmed, 2018; Gómez, Quintero, Salazar, 2019). Esta é 
considerada um material eficiente para ser hospedeiro de nanopartículas, atuando geralmente, em inúmeras áreas médicas (Ali; Ahmed, 2018). Segundo Shafabakhsh (2019), esse biomaterial é conhecido como um composto apropriado para a administração de quimio-drogas no tratamento do CA. Além disso, a administração transmucosa da droga é facilitada por meio de suas características mucoadesivas e catiônicas, aumentando a interação com a membrana mucosa.

Nesse sentido, em virtude da heterogeneidade, novas estratégias que abordam os processos de morte neoplásica são avaliadas, abrindo espaço para a utilização das nanopartículas que amenizam efeitos colaterais disseminados das quimioterapias por causa da sua especificidade direcionada ao tecido tumoral e da sua maior absorção (Lopes et al., 2019; Jiang et al., 2021). Haja vista, que a nanotecnologia é promissora e com múltiplas vantagens para saúde humana (Ali \& Ahmed, 2018). Portanto, a finalidade do presente estudo é realizar uma análise comparativa e crítica sobre a quimioterapia convencional e nanofibras de quitosana à frente do tratamento de tumores, confrontando seus benefícios e malefícios a saúde humana.

\section{Metodologia}

O presente artigo é um estudo qualitativo com caráter bibliográfico em fontes secundárias, trata-se de uma coleta de dados sobre análise comparativa e crítica sobre a quimioterapia convencional e nanofibras de quitosana à frente do tratamento de tumores, confrontando seus benefícios e malefícios a saúde humana. Empregaram-se nas buscas científicas artigos publicados nacionalmente e internacionalmente em diferentes bases de dados: Science Direct, PUBMED, Research, Societyand Development journal (RSDjournal), Scientific Electronic, Library Online (SCIELO), Governo do Brasil (gov.br).

Os critérios de inclusão sobre o referido levantamento deram-se através do uso das combinações de palavras-chaves em inglês "Chitosan; Nanofibers; Cancer; Chemotherapy; Advantage; Disadvantage", e as palavras em português "Sindrome de Moebius, Misoprostol, Aborto; Vantagem e Desvantagem". Com o intuito de tornar a busca mais específica, realizaram-se as conexões entre os termos com a utilização dos operadores booleanos "AND", que na integra retrata-se da temática referente confrontação entre a quimioterapia convencional e nanofibras de quitosana à como recurso terapêutico de neoplasias. Como critérios de exclusão eliminaram-se 474 artigos dos 504 encontrados. Neste estudo, as referências bibliográficas selecionadas e utilizadas na pesquisa foram publicadas e indexadas nos referidos bancos de dados dos anos de 2017 a 2022. Assim, nesse levantamento são reportados 11 do Science Direct, 14 da PUBMED, 1 da RSDjournal, 3 da SCIELO e 1 do gov.br, como demonstra-se no quadro a seguir (figura 1).

Neste estudo utilizou-se o total de XX artigos, limitando-se os resultados das buscas em inglês, espanhol e português. Todas as pesquisas, como a triagem de títulos, resumos e a seleção de estudos executaram-se pelos pesquisadores que trabalharam de forma independente, verificando a coerência e validação das informações do problema abordado. Em seguida das compilações resolveu-se as divergências por consenso em relação à duplicidade e as não relacionadas à temática principal. Assim, utilizaram-se todos os artigos considerados potencialmente legíveis para revisão do seu texto completo. Após isso, para a análise dos dados utilizou-se o programa Microsoft Excel versão Windows 10. 
Figura 1: Fluxograma com as etapas de análise realizadas para seleção dos artigos científicos.
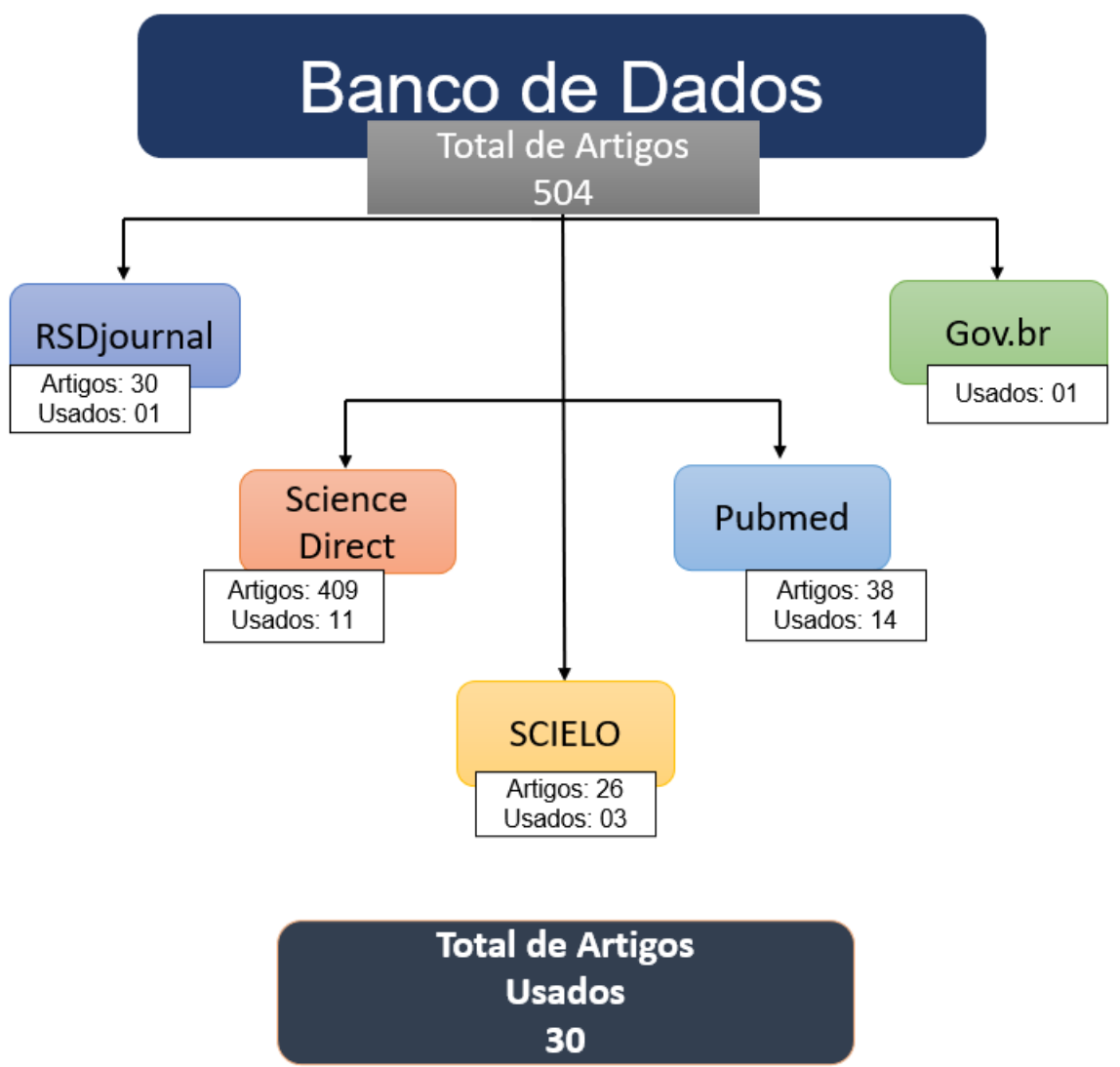

Fonte: Souza (2022).

A Quadro 1 apresenta a listagem das principais publicações encontradas na busca ativa nos bancos de dados de periódicos, acerca da temática "Análise comparativa entre a quimioterapia e nanofibras de quitosana à frente de neoplasias malignas".

Quadro 1: Artigos científicos listados dos bancos de dados utilizados no presente estudo.

\begin{tabular}{|l|c|c|}
\hline \multicolumn{1}{|c|}{ Título do artigo } & Autores e ano da pesquisa & Revista cientifica \\
\hline A review on chitosan and its nanocomposites in drug delivery & Ali, Ahmed, 2018 & Science Direct \\
\hline $\begin{array}{l}\text { Biomedical application of chitosan-based nanoscale delivery } \\
\text { systems: Potential usefulness in siRNA delivery for cancer } \\
\text { therapy. }\end{array}$ & Ashrafizadeh et al., 2021 & Science Direct \\
\hline $\begin{array}{l}\text { Nanotechnology-based drug delivery systems for the } \\
\text { improved sensitization of tamoxifen }\end{array}$ & Chao et al., 2021 & Science Direct \\
\hline $\begin{array}{l}\text { A study on structural comparisons of } \alpha \text {-chitin extracted from } \\
\text { marine crustacean shell waste. }\end{array}$ & Mohan et al., 2021 & Science Direct \\
\hline $\begin{array}{l}\text { Chitosan: A compound for drug delivery system in gastric } \\
\text { cancer-a review }\end{array}$ & Shafabakhsh et al., 2020 & Science Direct \\
\hline $\begin{array}{l}\text { Surface-deacetylated chitin nanofibers reinforced with a } \\
\text { sulfobutyl ether } \beta \text {-cyclodextrin gel loaded with prednisolone } \\
\text { as potential therapy for inflammatory bowel disease. }\end{array}$ & Tabuchi et al., 2017 & Science Direct \\
\hline $\begin{array}{l}\text { Applications of chitin and chitosan nanofibers in bone } \\
\text { regenerative engineering }\end{array}$ & Tao et al., 2020 & Science Direct \\
\hline $\begin{array}{l}\text { Electrospun polymeric nanofibers: New horizons in drug } \\
\text { delivery. }\end{array}$ & Thakkar, Misra, 2017 & Science Direct \\
\hline $\begin{array}{l}\text { drug self-delivery systems as a versatile nanoplatform for } \\
\text { cancer therapy: A review. }\end{array}$ & Xu et al., 2019 & S \\
\hline
\end{tabular}




\begin{tabular}{|c|c|c|}
\hline $\begin{array}{l}\text { Quimioterapia imunogênica: dependência de dose e esquema e } \\
\text { combinação com imunoterapia }\end{array}$ & Wu, Waxman,2018 & Science Direct \\
\hline $\begin{array}{l}\text { Chitosan/carbon quantum dot/aptamer complex as a potential } \\
\text { anticancer drug delivery syst0em towards the release of 5- } \\
\text { fluorouracil }\end{array}$ & Zavareh et al., 2020 & Science Direct \\
\hline Polytherapy and Targeted Cancer Drug Resistance & Chatterjee, Bivona, 2019 & Pubmed \\
\hline $\begin{array}{l}\text { Research Progress of Carrier-Free Antitumor Nanoparticles } \\
\text { Based on Phytochemicals }\end{array}$ & Jiang et al., 2021 & Pubmed \\
\hline $\begin{array}{l}\text { applications of chitosan electrospun nanofibers as a green } \\
\text { polymer-Review. }\end{array}$ & Kalantari et al., 2019 & Pubmed \\
\hline $\begin{array}{l}\text { Chitosan-based Polymer Matrix for Pharmaceutical Excipients } \\
\text { and Drug Delivery. }\end{array}$ & Khan et al., 2019 & Pubmed \\
\hline Natural products as multidrug resistance modulators in cancer & Kumar, Jaitak, 2019 & Pubmed \\
\hline $\begin{array}{l}\text { Quality of life of patients with cancer undergoing } \\
\text { chemotherapy in hospitals in Belo Horizonte, Minas Gerais } \\
\text { State, Brazil: does individual characteristics matter?. }\end{array}$ & Moreira et al., 2021 & Pubmed \\
\hline Chitosan oligosaccharide (COS): An overview. & Naveed et al., 2019 & Pubmed \\
\hline $\begin{array}{l}\text { Chitin and chitosan preparation from shrimp shells Penaeus } \\
\text { monodon and its human ovarian cancer cell line, PA-1. }\end{array}$ & $\begin{array}{l}\text { Srinivasan, Kanayairam, } \\
\text { Ravichandran, } 2018\end{array}$ & Pubmed \\
\hline $\begin{array}{lrrr}\begin{array}{l}\text { Recentprogress } \\
\text { enhancedcancercheoterapy }\end{array} & \text { nanomedicine } & \text { for } \\
\end{array}$ & Wei, 2021 & Pubmed \\
\hline $\begin{array}{l}\text { Sistemas de auto entrega de medicamentos para terapia do } \\
\text { câncer }\end{array}$ & Qin, 2017 & Pubmed \\
\hline O uso sustentável de polímeros & Silva, 2017 & Pubmed \\
\hline $\begin{array}{l}\text { Addis Abada population-basedipatternof câncer therapy, } \\
\text { Ethiopia }\end{array}$ & Feuchtne, 2019 & Pubmed \\
\hline $\begin{array}{l}\text { Extractionandcharacterizationofchitinscalesfromredtilapia } \\
\text { (oreochromis sp.) fromHuilaColombiabychemicalmethods }\end{array}$ & Gómez , 2019 & Pubmed \\
\hline $\begin{array}{l}\text { Extraction, characterization and antimicrobial activity of } \\
\text { chitosan from pen shell, Pinna bicolor }\end{array}$ & Sudatta et al., 2020 & Pubmed \\
\hline $\begin{array}{l}\text { A quimioterapia sob a ótica da pessoa com câncer : uma } \\
\text { análise estrutural }\end{array}$ & Wakiuchi, 2019 & Scielo \\
\hline $\begin{array}{l}\text { Qualidade de vida de idosos submetido a quimioterapia anti- } \\
\text { neoplásicos atendidos em um hospital de referência } \\
\text { oncológico }\end{array}$ & Souza, 2018 & Scielo \\
\hline $\begin{array}{l}\text { Construção e validação do histórico de enfermagem para } \\
\text { pacientes em quimioterapia }\end{array}$ & Lima, 2019 & Scielo \\
\hline $\begin{array}{l}\text { Eficácia da quimioterapia nos cuidados paliativos em } \\
\text { pacientes oncológicos: uma revisão integrativa }\end{array}$ & Carvalho et al., 2020 & RSDjournal \\
\hline $\begin{array}{l}\text { Avaliação da qualidade de vida de pacientes submetidos à } \\
\text { quimioterapia paliativa }\end{array}$ & Belmiro, 2018 & Gov.br \\
\hline
\end{tabular}

Fonte: Mendes (2022).

\section{Resultados e Discussão}

\subsection{Câncer}

Apesar dos avanços na saúde quanto ao tratamento das neoplasias, ainda é um estigma de óbito e, portanto, seu diagnóstico torna-se amedrontador, geralmente, acompanhado de angústia, desesperança, isolamento e temores (Sousa et al., 2018; Kumar \& Jaitak, 2019). Dentre as diversas formas de procedimentos para o tratamento oncológico, as mais utilizadas são a cirurgia, a radioterapia e a quimioterapia, que são definidas de acordo com as características, a extensão e o tipo histológico do tumor, com a opção por uma modalidade de tratamento isolada ou de forma combinada (Moreira et al., 2021).

“O câncer é um termo que abrange mais de 100 diferentes tipos de doenças malignas que têm em comum o crescimento desordenado de células, que podem invadir tecidos adjacentes ou órgãos à distância. Dividindo-se rapidamente, estas células tendem a ser muito agressivas e incontroláveis, determinando a formação de tumores, que podem espalhar-se para outras regiões do corpo. Os diferentes tipos de CA correspondem aos vários tipos de células do corpo, ou seja, quando começam em tecidos epiteliais, como pele ou mucosas, são denominados carcinomas. Se o ponto de partida são os tecidos conjuntivos, como osso, músculo ou cartilagem, são chamados sarcomas" (Instituto Nacional de Câncer - INCA, 2020). 
Ressalta-se que, os eivados padecem com impacto, vivenciando reações adversas corroboradas pelas drogas. Com isso, apesar das estratégias de controle desses efeitos, há relevantes motivos para não realização ou descontinuação do tratamento (Feuchtner et al., 2019). À vista disso, faz-se importante atentar-se para a avaliação e inclusão de alternativas inovadoras nos serviços de saúde que diminuam os impactos provocados pelos principais tratamentos oncológicos utilizados nesse recorte geográfico.

\subsection{Quimioterapia}

Diversas substâncias foram descobertas ou sintetizadas desde a Segunda Guerra Mundial. A quimioterapia, uma dessas substâncias, é atualmente um componente crucial dos tratamentos de CA para vários tipos, estabelecendo estratégias de empregar fármacos tóxicos, no intuito de causar apoptose das células cancerígenas (Wey et al., 2021).

A quimioterapia é muito utilizada em pacientes com tumores incuráveis, onde são tratados por uma equipe multidisciplinar responsável para aliviar e minimizar os danos causados pelo tumor. Logo, tem como objetivo aumentar a sobrevivência do paciente, mas sem intenção de curar (Carvalho et al., 2020). Por conseguinte, sua aplicação convencional, como doxorrubicina, mitoxantrona e ciclofosfamida, corroborá para ativação da morte celular imunogênica (ICD), ou seja, interagem com o sistema inato e adaptativo, induzindo assim, a um sinal da célula maligna para serem deglutinadas, capturam os antígenos relacionados a tumor, os lisossomos liberam seu ATP, eleva produção de macrófagos, entre outras (Wu \& Waxman, 2018).

Um grande empecilho é a resistência a múltiplas drogas (MDR), refere-se a um fenótipo e as células cancerosas que inicialmente era sensível tornam-se resistentes a diferentes drogas com estruturas e semelhanças moleculares variadas (Wey et al., 2021; Kumar \& Jaitak, 2019). Diante isso, o CA procura alternativas que o fármaco em doses relevantes não obterá o mesmo efeito, sejam por resistência intrínseca ou adquirida, alterando seu receptor alvo, adaptações, mudanças epigéneticas, downstream (mudança de curso) (Chatterjee \& Bivona, 2019; Kumar \& Jaitak, 2019).

Apesar de obterem grandes avanços, o CA continua ser a prior dos acometimentos com risco de vida em todo o mundo, contendo, por volta de, 18,1 milhões de novos casos e 9,6 milhões de óbitos pela mesma ocorreram em 2018 (Bray et $a l$, 2018). Dessa forma, avanços para abordar esses problemas geralmente se relacionam com o emprego de nanocarreadores assistentes, como nanoarquiteturas automontadas e nanofibras inorgânicos para entrega de drogas, que protegem as drogas da rápida depuração sanguínea/renal e atingem o acúmulo preferencial de drogas dentro de tumores sólidos devido ao efeito de permeabilidade e retenção aumentada (EPR). Esses sistemas de liberação de drogas (DDSs) são realmente eficazes e alguns deles foram submetidos a ensaios clínicos (Xue et al., 2019).

\subsection{Quitina}

A quitina é um polímero de alto peso molecular, que se compõe de exoesqueletos à paredes celulares fungicas, são encontrados de diversificadas formas cristalinas, conhecidas como $\alpha, \beta$ e $\gamma$. A forma $\alpha$-quitina é a mais estável entre elas, por possuir forma antiparalela das suas cadeias de polissacarídeo, ademais se localiza em cascas de camarões e caranguejo. $\mathrm{Na}$ forma $\beta$ - quitina se acha em canetas feitas por tinta de lulas, no entanto, é constituída por forças intermoleculares mais frágeis que a $\alpha$. (Sudatta et al., 2020; Knidri et al., 2018).

A quitina é um polissacarídeo natural apreciada por sua biocompatibilidade, biodegradabilidade, atóxica, com particularidades de ser quelante, emulsificante e antimicrobiana. Além dos seus aspectos físico-químicos sucedem diversas propriedades como, a capacidade filmogênica, a acessibilidade na formação de géis e a resistência mecânica que proporciona o interesse dos campos de agroindústria, farmacêutica e biotecnologia (Srinivasan et al., 2018). 
Suas particularidades são aplicadas na biomedicina, conseguindo facilmente ser moldável nas formas de géis, membranas, micropartículas, suporte, esponjas, entre diversas outras. Contudo, a forma mais relevante para a entrega de fármacos contra o CA trabalhados no campo de nanobiotecnologia, é por nanofibras a base de quitina ou quitosana como membrana e andaimes de nanocompósitos ou nanopartículas (Kalantari et al., 2019).

\subsection{Extrações da quitina}

Na extração da quitina, é realizada por um processo químico, a desmineralização da casca dos crustáceos, dissolvendo em ácidos e bases fortes, para a desproteinização, com intuito, de retirar as proteínas e a calcita $\left(\mathrm{CaC}^{3}\right)$. O tempo utilizado na realização da extração e a concentração dos ácidos se devem a fonte de quitina, com isso, o polímero degrada-se em elevadas temperaturas (Mohan et al., 2021).

A extração é executada com ácido clorídrico $(\mathrm{HCl})$ para a desmineralização, em seguida, filtra-se com água destilada até o pH torna-se neutro. Logo após, ocorre a desproteinização, utilizando hidróxido de sódio $(\mathrm{NaOH})$, clorofórmio, metanol $\left(\mathrm{CH}^{3} \mathrm{OH}\right)$ e água destilada, resultando descolorimento e formando o produto desejado (Mohan et al., 2021).

\subsection{Quitosana}

A desacetilação parcial da quitina produz quitosana, sendo este um polímero formado por composto de N-acetil-Dglucosamina, D-glucosamina, um grupo amino $\left(\mathrm{NH}^{2}\right)$ e dois grupos de hidroxila $(\mathrm{OH})$, seu peso molecular consiste em 5,3 x $10^{5}$, constituído de $44,1 \%$ de carbono, $6,84 \%$ de hidrogênio e 7,97\% nitrogênio. Dissolve-se em soluções alcalinas, mas preferível em solubilidades ácidas (Ashrafizadeh et al., 2021). Nesse sentido, é geralmente aplicada como biomaterial, especialmente para DDs e uso em combinação com outras substâncias para melhorar seus efeitos terapêuticos. (Satitsri \& Muanprasat, 2020).

A quitosana refere-se a um dos derivados poliméricos de quitina solúveis, que são descritos e classificados de acordo com várias características onde o grau de polimerização (DP) e a fração de resíduos N -acetilados ( FA ) são os mais importantes (Harmsen et al, 2019).Esse polímero apresenta atributos biológicos notórios pela relevância na formação de biomateriais, contendo funcionalidades, no sistema de entrega de drogas, por demonstra propriedades biocompatíveis, biodegradáveis, hemostasticas, bacteriostática, anticarcinogênica e fungistática (Naveed et al., 2019).Diversas outras particularidades desse biomaterial são transfecção, gelificação in situ, inibição na bomba de efluxo, liberação controlada de droga, mucoadesão, entre outras. Outrossim, o método de gelificaçao proporciona um material promissor para ser sintetizado em nanoescala (Ali, Ahmed, 2018; Ashrafizadeh et al., 2021).

Ademais, todas essas características devem-se ao seu grupo funcional de amina primária e a sua bioadesividade é uma particularidade que possibiliza a aderência do biopolímero em tecidos brandos ou rígido, sendo utilizados nos campos de odontologia, oftalmologia, ortopedia, medidas cirúrgicas e biomédicas (Ali, Ahmed, 2018; Naveed et al., 2019; Ashrafizadeh et al., 2021).

Além disso, a quitosana é de natureza catiônica e sua solubilidade em água é baixa, mas é solúvel em soluções de baixo $\mathrm{Ph}$. Dessa forma, diferentes derivados da quitosana são produzidos para superar essa limitação para fins de entrega controlada de substâncias. Por esse motivo é aproveitada para interação eletrostática com ácidos nucleicos, e a mesma tem sido usada como transportador de entrega de genes para terapia de câncer e aplicação como um potencial imunoadjuvante para vacinas contra o câncer (Khan et al., 2019).

Assim, a nanotecnologia ou biotecnologia, oferece grandes vantagens para a saúde humana, por ser uma tecnologia promitente, como o DDs de medicamentos controlados, considerado promissor pela sua especificidade, com intuito de 
convergir-se a quantidade de agentes quimioterápicos, apenas para a área afetada em especifico, evitando a distribuição sistêmica, que ocasiona efeitos colaterais (Kalantari et al., 2019; Ali, Ahmed, 2018).

\subsection{Extração da quitosana}

A CS consegue ser extraída de duas formas, pelo método químico, que consiste no processo de desmineralização, descoloração, desacetilação, desproteinização, para obter dos crustáceos, este método comumente utilizado, pela sua acessibilidade, benefício e larga produção. Dessa maneira, as ligações de N-acetil da quitina são lisadas concebendo a Dglicosamina, que contêm um grupo amino livre. Quando o polímerodemonstra-se solúvel em meio aquoso forma-se a CS,ou seja, quando a quantidade de D-glicosamina for similar ou superior a 50\% no grau de desacetilação (Madni et al., 2021; Shafabakhsh et al., 2020)

Nesse procedimento, são desmineralizados das conchas com cloreto de hidrogênio $(\mathrm{HCl})$ e desproteinização com hidróxido de sódio $(\mathrm{NaOH})$ para retirar os resíduos de proteínas e minerais, por seguinte, emprega-se oxido de potássio $\left(\mathrm{K}^{2} \mathrm{O}\right)$ e manganês $(\mathrm{Mg})$ para retirar moléculas pigmentadas, advindo a quitina transparente e submetendo a um alcalino forte para a formação da CS (Madni et al., 2021).

O outro método é o enzimático, por uma enzima denominada quitina desacetilase, obtida por bactérias, fungos e outras espécies de insetos. Onde ocorre a hidrolise da ligação $\mathrm{N}$-acetil-amida, que contêm na sua molécula, quando ocorre essa lise, a mesma é modificada quimicamente, produzindo-se a reprecipitação, glicolação e despolimerização logo na sua forma bruta são uma substância escassa de enzimas (Madni et al., 2021; Zhu et al., 2019).

\subsection{Nanofibras}

Uma nova área de pesquisa multidisciplinar, que engloba células vivas, manuseio por meio de ambiente genético ou extracelular, otimização de implantações no corpo por substitutos biológicos e proporcionamento a renovação de tecidos de maneiras ativas, referem-se ao campo de engenharia essas áreas. O propósito da utilização das fibras nessa área deve-se ao intuito de converter, restaurar, preservar ou aprimorar a função de um determinado tecido ou órgão (Ahmad et al., 2020).

Uma técnica inovadora e simplificada, constituindo pela eletrofiação de polímeros em fibras ultrafinas com nano dimensão, conhecida por nanofibras, é uma modernidade com promissão para entrega de medicamentos, contanto que suas variáveis de processamento - no período da fabricação - são controladas com precisão (Ali, Ahmed, 2018).

Os quesitos básicos para construir um andaimes de polímero na bioengenharia, como as nanofibras, é essencial conter alta porosidade, com disposição de poros adequados, uma ampla dimensão de superfície, biodegradabilidade, com o índice de degradação proporcional à formação de neotecido e o andaime dispõe de integridade estrutural fundamental para prevenir que os poros dele, possam colapsas, no decorrer da sua composição, mesmo com as propriedades mecânicas apropriada (Ahmad $e t$ al., 2020)

O polissacarídeo natural, quitosana, é utilizado em procedimentos biológicos por décadas e atualmente empregado como nanocompósitos. Esses apontam bons atributos mecânicos e estabilidade térmica, dessa forma, podem encontra-se em diversos formatos, como em filme, malhas de filha, pó, grânulos, membranas, hidrogeis, nanofibras, entre outras, segundo a sua aplicabilidade (Ali \& Ahmed, 2018).

Outrossim, as fibras manométricas de CS produzidas pelo método de eletrofiação permite ser alterada por modificação quimicamente, os grupos amino ou criando interações eletrostáticas entre os grupos amina (cátion) a que fica na superfície e os demais componentes de carga negativas (ânion), favorecendo assim de forma física, química e biológica (Tabuchi et al., 2017; Thakkar \& Misra, 2017). 
A eletrofiação é uma inovação técnica para a construção de fibras de polímeros em nano escalas, contendo benefício por ser um método simples, fácil e econômico, ou seja, com custo-benefício na fabricação, incorpora o bioativo nas nanofibras, facilmente, além de não necessitar do calor no decorrer do processo, isso é relevante, logo alguns compostos são sensíveis a temperatura (Kalantari et al., 2019). As fibras de CS são combinadas com outros polímeros para modificar o aspecto biológico e mecânico dos andaimes, independentemente, da produção por eletrofiação, autopolimerização e termicamente induzidas por separação de fases (Tao et al., 2020).

\subsection{Entrega de drogas}

O CA ainda é um grande problema na saúde pública e com a resistência das células tumorais, o desafio consiste na terapia com drogas sintéticas, que estão perdendo a eficácia (Ashrafizadeh et al., 2021). Com a evolução da nanotecnologia, modernizaram a entrega de drogas por vias orais, tópicas e parentais, como as nanopartículas que potencializam o aumento da biodisponibilidade, especificidade, eficácia e adesão da terapia medicamentosa (CHAO et al., 2020). O quimioterápico mais utilizado e eficiente é o 5-flurouracil (5-FU), para inibir o aumento de células tumorais e agressivas, atuando na inibição do timidilatosintase (Zhu et al., 2019).

A quimioterapia tornou-se uma tática predominante para as majoritárias terapias, devido à sua alta eficiência em comparação com outras, antes existentes (Jiang et al., 2021). No entanto, gerando o questionamento dos pesquisadores da prescrição do tratamento, por possui muitos efeitos colaterais intensos, devido ao potencial promotor de apoptose, nas células cancerígenas e nas células normais, assim não se tornando um tratamento benéfico para muitos pacientes (Carvalho et al., 2020; Belmiro, 2018).

Ademais, os esses agentes convencionais apresentam limitações inerentes, como distribuição inespecífica, baixa biodisponibilidade, rápida depuração sanguínea e redução da solubilidade em ambientes fisiológicos (Jiang et al., 2021). Contudo, essa é reconhecida pelos efeitos colaterais que acometem os pacientes durante o período de aplicação, correspondendo de 50\% à 80\% dos pacientes tratados com esse medicamento, consiste em perda de cabelo, mal estar, náuseas, vômitos, alopecia e, principalmente, mucosite intestinal, levando a graves diarreias. Com isso, requer a redução da dosagem ou descontinuo do tratamento, limitando o êxito da terapia (Koizumi et al., 2017; Jiang et al., 2021).

Associadas a isso, há alterações na aparência física, dificuldade para manter um vínculo empregatício, as relações interpessoais e os questionamentos sobre a possibilidade de cura, prejudicando, igualmente, os âmbitos psicossociais (Bushatsky et al.,2017). Entretanto, é essencial projetar uma terapia direcionada, que alcance, apenas, o gene responsável pela malignidade e crescimento do CA (Ashrafizadeh et al., 2021).

Um dos problemas mais pertinentes dos quimioterápicos na aplicação, é a cobiça para descobrir uma entrega de fármacos que solucione a tolerância de $\mathrm{pH}$, baixa solubilidade em água e difusão intracelular. A quitosana demonstra-se ser adequado para esse sistema de entregas em diferentes tipos de tumores (Shafabakhsh et al., 2020).

A solubilidade da quitosana resulta na protonação em meios ácidos, logo contém um polímero catiônico que possibilita que interaja com diversas moléculas, que auxilia na DDs, devido às condições fisiológicas. Os derivados de quitosana conseguem ser implantado na superfície de microesferas sílicas, por meio da combinação dos grupos amino $\left(\mathrm{NH}^{2}\right)$ protonado em ambientes levemente ácidos, onde o $\mathrm{pH}$ é similar ao tumor da matriz celular. Ademais, conseguem ser empregues na entrega de drogas hidrofílicas e hidrofóbicas (Shafabakhsh et al., 2020).

Todavia, para tornar-se eficaz contra o CA, o tamanho da partícula é um fator determinante, pois quanto menor o tamanho melhor sua afinidade com as células. Os materiais em nanoescalas a base de CS, possuem alta estabilidade em locais com tumores, por representa-se tipicamente pelo seu $\mathrm{pH}$ ácido moderado, contribuindo para a entrega de drogas 
anticarcinogênica, conseguindoutilizar como guia durante a cirurgia de neoplasia maligna e encapsular materiais genéticos devido à carga negativa (Ashrafizadeh et al., 2021).

A CS demonstra-se biocompatível e possui a capacidade de liberação sustentada dos compostos anticancerígenos, por conseguir carregar fármacos com o $\mathrm{IC}^{50}$ reduzidos e agentes sintéticos, como 5-FU, aumentando a disponibilidade e eficácia contra o CA (Zavareh et al.,2020). Além disso, dois fatores importantes nessa atividade, são o tamanho - nano partículas de quitosana- e a estabilidade em locais do tumor, que possui grande afinidade com seu pH (Ashrafizadeh et al., 2021).

Os receptores específicos na superfície celular conseguem interagir com os ligantes específicos em nanopartículas de CS, ocorrendo a endocitose. Os receptores mais frequentes para manipulação dos fármacos anticarcinogenicos são receptores de folatos, receptor de CD44, receptores de lipoproteínas de baixa densidade e integrinas e receptor epidérmico do fator de crescimento (EGFR) (Shafabakhsh et al., 2020).

Contudo, os endolisossomos nas células não conseguem degradar as nanopartículas de CS transportadas por drogas, elevando sua concentração intracelular, por seguinte liberando-as gradualmente pelas nanopartículas. Essa projeção é relevante a quantidade de receptores expressos em qualquer categoria de células cancerosas (Shafabakhsh et al., 2020).

\section{Conclusão}

Este estudo proporcionou avaliar um comparativo entre o tratamento quimioterápico padrão e a utilização de nanofibras de quitosana. Obtendo resultados favoráveis ao procedimento alternativo, haja vista, os efeitos colaterais adversos da terapia convencional, evidenciando a necessidade de novas medidas para aprimora-ló. Ademais, um sistema de entrega de drogas direcionadas por nanofibras de quitosana, contendo em vista, a sua biocompatibilidade e capacidade de transportar fármacos e agentes sintéticos de maneira eficiente, faz-se necessário e essencial para a redução dos danos físicos e emocionais ao paciente. Com isso, é primordial explorar e desenvolver métodos mais simples e bem aplicados para o CA contém grande relevância na pesquisa e valor clínico.

\section{Referências}

Ali, A. \& Ahmed, S. (2018). A review on chitosan and its nanocomposites in drug delivery. International Journal of Biological Macromolecules, 109, 273286. 10.1016/j.ijbiomac.2017.12.078

Ashrafizadeh M. Delfi, M. Hashemi, F. Zabolian, A. Saleki, H. Bagherian, M. Azami, N. Farahani, M. V. Sharifzadeh S. O. Hamzehlou, S. Hushmandi, K. Makvandi, P. Zarrabi A. Hamblin, M. R. \& Varma, R. S. (2021). Biomedical application of chitosan-based nanoscale delivery systems: Potential usefulness in siRNA delivery for cancer therapy. Carbohydrate Polymers, 260, 117809, 10.1016/j.carbpol.2021.117809.

Belmiro, A. A. M. D. L. M. (2018). Avaliação da qualidade de vida de pacientes submetidos à quimioterapia paliativa. Dissertação (Mestrado em Enfermagem), Universidade de Brasília. https://repositorio.unb.br/handle/10482/34176.

Carvalho, T. V., Nakashima, S. S., Correia, T. L. B. V., Dias, S. B., Silva, M. A., dos Santos, R. C., \& Pena, H. P. (2020). Eficácia da quimioterapia nos cuidados paliativos em pacientes oncológicos: uma revisão integrativa. Research, Societyand Development, 9(11), 2525-3409. $10.33448 /$ rsd-v9i11. 10267.

Chatterjee N., \& Bivona T. G. (2019). Polytherapy and Targeted Cancer Drug Resistance. Trends Cancer. 5(3):170-182. 10.1016/j.trecan.2019.02.003.

Chao, X. Zhao, L. Mou, Y. \& Zhang, P. (2021). Nanotechnology-based drug delivery systems for the improved sensitization of tamoxifen. Journal of Drug Delivery Science and Technology. 61, 102229. 10.1016/j.jddst.2020.102229.

Feuchtner J, Mathewos A, Solomon A, Timotewos G, Aynalem A, \& Wondemagegnehu T. (2019). Padrão de terapia do câncer baseado na população de Adis Abeba, Etiópia. 10.1371/journal.pone.0219519

García Gómez, A. G. Conde Quintero, M. \& Castro Salazar, H. T. (2019). Extração e caracterização de escamas de quitina de tilápia vermelha (oreochromis sp.) de Huila, Colômbia por métodos químicos. Revista Ingenierías Universidad De Medellín, 18 (34), 71-81. 10.22395/rium.v18n34a5

Jiang, S., Fu, Y., Zhang, X., Yu, T., Lu, B., \& Du, J. (2021). Research Progress of Carrier-Free Antitumor Nanoparticles Based on Phytochemicals. Frontiers in bioengineering and biotechnology, 9, 799806. 10.3389/fbioe.2021.799806

Kalantari, K., Afifi, A. M., Jahangirian, H., \& Webster, T. J.(2019). Biomedical applications of chitosan electrospun nanofibers as a green polymer - Review. Carbohydrate Polymers, 207, 588-600. 10.1016/j.carbpol.2018.12.011 
Khan M. I. H., An X., Dai L., Li H., Khan A., \& Ni Y. (2019). Chitosan-based Polymer Matrix for Pharmaceutical Excipients and Drug Delivery. Curr Med Chem. 26(14):2502-2513. 10.2174/0929867325666180927100817.

Kumar A., \& Jaitak V. Natural products as multidrug resistance modulators in cancer. Eur J Med Chem. 15, 176:268-291. 10.1016/j.ejmech.2019.05.027.

Lima, M. T. (2019). Construção e validação do histórico de enfermagem para pacientes em quimioterapia.RevBrasEnferm. 72(2):409-17. 10.1590/0034-71672018-0031

Mohan, K., Muralisankar, T., Jayakumar, R., \& Rajeevgandhi, C. ( 2021). A study on structural comparisons of $\alpha$-chitin extracted from marine crustacean shell waste. Carbohydrate Polymer Technologies and Applications, 2, 100037, 2021. 10.1016/j.carpta.2021.100037

Moreira, D. P. Simino, P. R Reis, I. A. Santos, M. A. C. \& Cherchiglia, M. L. (2021). Quality of life of patients with cancer undergoing chemotherapy in hospitals in Belo Horizonte, Minas Gerais State, Brazil: does individual characteristics matter? Cadernos de SaúdePública. 37(8). 10.1590/0102$311 \mathrm{X} 00002220$

Naveed M, Phil L, Sohail M, Hasnat M, Baig MMFA, Ihsan A. U, Shumzaid M, Kakar M. U, Mehmood Khan T, Akabar M. D, Hussain M. I, \& Zhou Q. G. (2019). Chitosan oligosaccharide (COS): An overview. International journal of biological macromolecules, 129, 827-843. 10.1016/j.ijbiomac.2019.01.192

Qin S. Y, Zhang A. Q, Cheng S. X, Rong L., \& Zhang X. Z. (2017). Drug self-delivery systems for cancer therapy. Biomaterials. Jan;112:234-247. 10.1016/j.biomaterials.2016.10.016.

Shafabakhsh, R. Yousefi, B. Asemi, Z. Nikfar, B. Mansournia, M. A. \& Hallajzadeh, J.(2020). Chitosan: A compound for drug delivery system in gastric cancer-a review. Carbohydrate Polymers, 242, 10.1016/j.carbpol.2020.116403

Silva, F. A. \& Rabelo, D. (2017). O Uso Sustentável de Polímeros. Revista Processos Químicos, 11(21), 10.19142/rpq.v11i21.387

Souza, J. C. Santos, E. G. A. Santos, A. L. S. Santos, M. I. P. O. Fernandes, D. S. \& Oliveira, T. N. C. (2018). Qualidade de vida de idosos submetidos à quimioterapia antineoplásica atendidos em um hospital de referência oncológica. Rev Pan-Amaz. Saude. 9(3):47-55. 10.5123/S2176-62232018000300006

Srinivasan, H. Kanayairam, V. \& Ravichandran, R. (2018). Chitin and chitosan preparation from shrimp shells Penaeus monodon and its human ovarian cancer cell line, PA-1. International journal of biological macromolecules, 107, 662-667. 10.1016/j.ijbiomac.2017.09.035.

Sudatta, B. P. Sugumar V, Varma R, Nigariga P. (2020). Extraction, characterization and antimicrobial activity of chitosan from pen shell, Pinna bicolor. International Journal of Biological Macromolecules, 163, 423-430. 10.1016/j.ijbiomac.2020.06.291.

Tabuchi R, Anraku M, Iohara D, Ishiguro T, Ifuku S, Nagae T, Uekama K, Okazaki S, Takeshita K, Otagiri M, \& Hirayama F. (2017). Surface-deacetylated chitin nanofibers reinforced with a sulfobutyl ether $\beta$-cyclodextrin gel loaded with prednisolone as potential therapy for inflammatory bowel disease. Carbohydrate Polymers, 174, 1087-1094, 2017. 10.1016/j.carbpol.2017.07.028

Tao, F. Cheng Y, Shi X, Zheng H, Du Y, Xiang W, \& Deng H. (2020). Applications of chitin and chitosan nanofibers in bone regenerative engineering. Carbohydrate Polymers, 230, 115658. 10.1016/j.carbpol.2019.115658

Thakkar, S. \& Misra, M. (2017). Electrospun polymeric nanofibers: New horizons in drug delivery. European Journal of Pharmaceutical Sciences, 107, 148167. 10.1016/j.ejps.2017.07.001.

Xue P., Wang J, Han X, \& Wang Y. (2019). Hydrophobic drug self-delivery systems as a versatile nanoplatform for cancer therapy: A review. Colloids Surf B Biointerfaces. 1;180, 202-211. 10.1016/j.colsurfb.2019.04.050.

Wakiuchi, J., Marcon Silva, S., Oliveira, D. C. \& Sales Aparecida, C. (2019). A quimioterapia sob a ótica da pessoa com câncer: uma análise estrutural. Tese da Representações Sociais do câncer e da quimioterapia para pessoas adoecidas. Programa de Pós-Graduação em Enfermagem, Universidade Estadual de Maringá. 10.1590/1980-265X-TCE-2018-0025

Wei G, Wang Y, Yang G, Wang Y, \& Ju R. (2021). Progressos recentes em nanomedicina para quimioterapia avançada contra o câncer. Teranóstica; 11(13):6370-6392. 10.7150/thno.57828.

Wu, J., \& Waxman, D. J (2018). Quimioterapia imunogênica: dependência de dose e esquema e combinação com imunoterapia. Cartas de câncer, 419, 210221. 10.1016/j.canlet.2018.01.050

Zavareh, H. S. Pourmadadi, M. Moradi, A. Yazdian, F. \& Omidi, M . (2020). Chitosan/carbon quantum dot/aptamer complex as a potential anticancer drug delivery syst0em towards the release of 5-fluorouracil. International Journal of Biological Macromolecules, 165, 1422-1430, 10.1016/j.ijbiomac.2020.09.166. 